\title{
Paroxysmal nocturnal hemoglobinuria in systemic lupus erythematosus: a case report
}

\author{
Norio Nakamura ${ }^{1 *}$, Toshiyuki Sugawara ${ }^{2}$, Ken-ichi Shirato ${ }^{2}$, Ryuichiro Kumasaka ${ }^{2}$, Masayuki Nakamura², \\ Michiko Shimada², Takeshi Fujita², Reiichi Murakami², Yuko Shimaya², Hiroshi Osawa², Hideaki Yamabe² and \\ Ken Okumura ${ }^{2}$
}

\begin{abstract}
Introduction: Paroxysmal nocturnal hemoglobinuria is an acquired disorder of hemopoiesis and is characterized by recurrent episodes of intravascular hemolysis due to an increased sensitivity to complement-mediated hemolysis. Systemic lupus erythematosus with paroxysmal nocturnal hemoglobinuria is very rare. We report a case of paroxysmal nocturnal hemoglobinuria that developed in a patient with systemic lupus erythematosus and lupus nephritis.
\end{abstract}

Case presentation: A 29-year-old Mongolian woman had systemic lupus erythematosus, which manifested only as skin lesions when she was 12 years old. She had leg edema and proteinuria when she was 23 years old, and a renal biopsy revealed lupus nephritis (World Health Organization type IV). She had been treated with steroids and immunosuppressant therapy. At 29, she had headaches, nausea, general fatigue, and severe pancytopenia and was admitted to our hospital. A laboratory evaluation showed hemolytic anemia. Further examination showed a neutrophil alkaline phosphatase score of 46 points, a CD55 value of $18 \%$, and a CD59 value of $78.6 \%$. The results of Ham test and sugar water tests were positive. The constellation of symptoms throughout the clinical course and the laboratory findings suggested paroxysmal nocturnal hemoglobinuria.

Conclusions: To the best of our knowledge, systemic lupus erythematosus with paroxysmal nocturnal hemoglobinuria is very rare. Clinicians should be aware of the association between autoimmune and hematological diseases.

\section{Introduction}

Paroxysmal nocturnal hemoglobinuria $(\mathrm{PNH})$ is an acquired disorder of hemopoiesis and is characterized by recurrent episodes of intravascular hemolysis due to an increased sensitivity to complement-mediated hemolysis [1]. Systemic lupus erythematosus (SLE) with PNH is very rare. We present a case of PNH that developed in a 29-year-old woman who had SLE.

\section{Case presentation}

A 29-year-old Mongolian woman had SLE, which manifested only as skin lesions when she was 12 years old. Because she had leg edema and proteinuria with serological and hematological abnormalities - the titers of

\footnotetext{
* Correspondence: nnakamur@r2.dion.ne.jp

'Community Medicine, Hirosaki University Graduate School of Medicine, 5

Zaifu-cho, Hirosaki-city, Aomori, 036-8562, Japan

Full list of author information is available at the end of the article
}

anti-nuclear antibody and double-stranded DNA (dsDNA) antibody were increased and the lymphocyte count was decreased - at 23 years old, a renal biopsy was performed. The results revealed lupus nephritis (World Health Organization type IV). Her condition was diagnosed as SLE according to the criteria of the American College of Rheumatology [2]. She had been treated with steroids and immunosuppressants, including cyclophosphamide. She had pancytopenia at 25 years old, and secondary aplastic anemia, probably due to cyclophosphamide, was diagnosed. Pancytopenia was worsening six months after the onset of pancytopenia and therefore cyclosporine A was administered. When she was 28 years old, rheumatoid arthritis was diagnosed because of polyarthralgia and morning stiffness. After 2 months, she had severe headaches, and cerebral venous thrombosis was diagnosed by computed tomography. Laboratory data showed a high level of lactate

\section{() Biomed Central}


dehydrogenase (LDH), a low level of haptoglobin, and a negative Coombs test result. These results suggested that she had hemolytic anemia, and the dose of steroid was increased. Her condition improved gradually.

When she was 29 years old, she had headaches, nausea, general fatigue, and severe pancytopenia and was admitted to our hospital. A laboratory evaluation showed the following: hemoglobin of $7.3 \mathrm{~g} / \mathrm{dL}$, white blood cell count of $11,400 / \mu \mathrm{L}$, platelets of $4.2 \times 10^{4} / \mu \mathrm{L}$, total protein of $4.9 \mathrm{~g} / \mathrm{dL}$, albumin of $2.3 \mathrm{~g} / \mathrm{dL}, \mathrm{LDH}$ of $1085 \mathrm{U} / \mathrm{L}$, total bilirubin of $1.6 \mathrm{mg} / \mathrm{dL}$, blood urea nitrogen of $34 \mathrm{mg} / \mathrm{dL}$, creatinine of $1.1 \mathrm{mg} / \mathrm{dL}, C$-reactive protein of $11.5 \mathrm{mg} / \mathrm{dL}$, haptoglobin of less than $6 \mathrm{mg} /$ $\mathrm{dL}$, and dsDNA antibody of $5 \mathrm{IU} / \mathrm{L}$. The results of direct and indirect Coombs tests were negative. Further examination showed a neutrophil alkaline phosphatase score of 46 points, a CD55 value of $18 \%$, and a CD59 value of $78.6 \%$. The results of Ham test and sugar water tests were positive. Her urine was red because of hemolysis (Figure 1). The constellation of symptoms throughout the clinical course and the laboratory findings suggested $\mathrm{PNH}$.

\section{Discussion}

$\mathrm{PNH}$ is an acquired disorder of hemopoiesis and is characterized by recurrent episodes of intravascular hemolysis due to an increased sensitivity to complement-mediated hemolysis [1]. A crucial pathophysiological mechanism is an acquired defect of the glycosylphosphatidylinositol-anchored proteins, namely CD55 and CD59 [3].

Flow cytometry analysis of red blood cells with monoclonal antibodies directed against CD55 and CD59 is now the gold standard technique for the diagnosis of PNH [4]. The normal values of CD55 and CD59 are more than $85.4 \%$ and more than $99.8 \%$, respectively. In the present case, the values of CD55 and CD59 were $18 \%$ and $78.6 \%$, respectively. Consequently, PNH was diagnosed.

PNH presents three clinical manifestations: (a) an acquired intravascular hemolytic anemia due to the increased susceptibility of the erythrocyte membrane to complement-mediated lysis; (b) thrombosis in large vessels, such as hepatic, abdominal, cerebral, and subdermal veins; and (c) mild to severe bone marrow hypoplasia

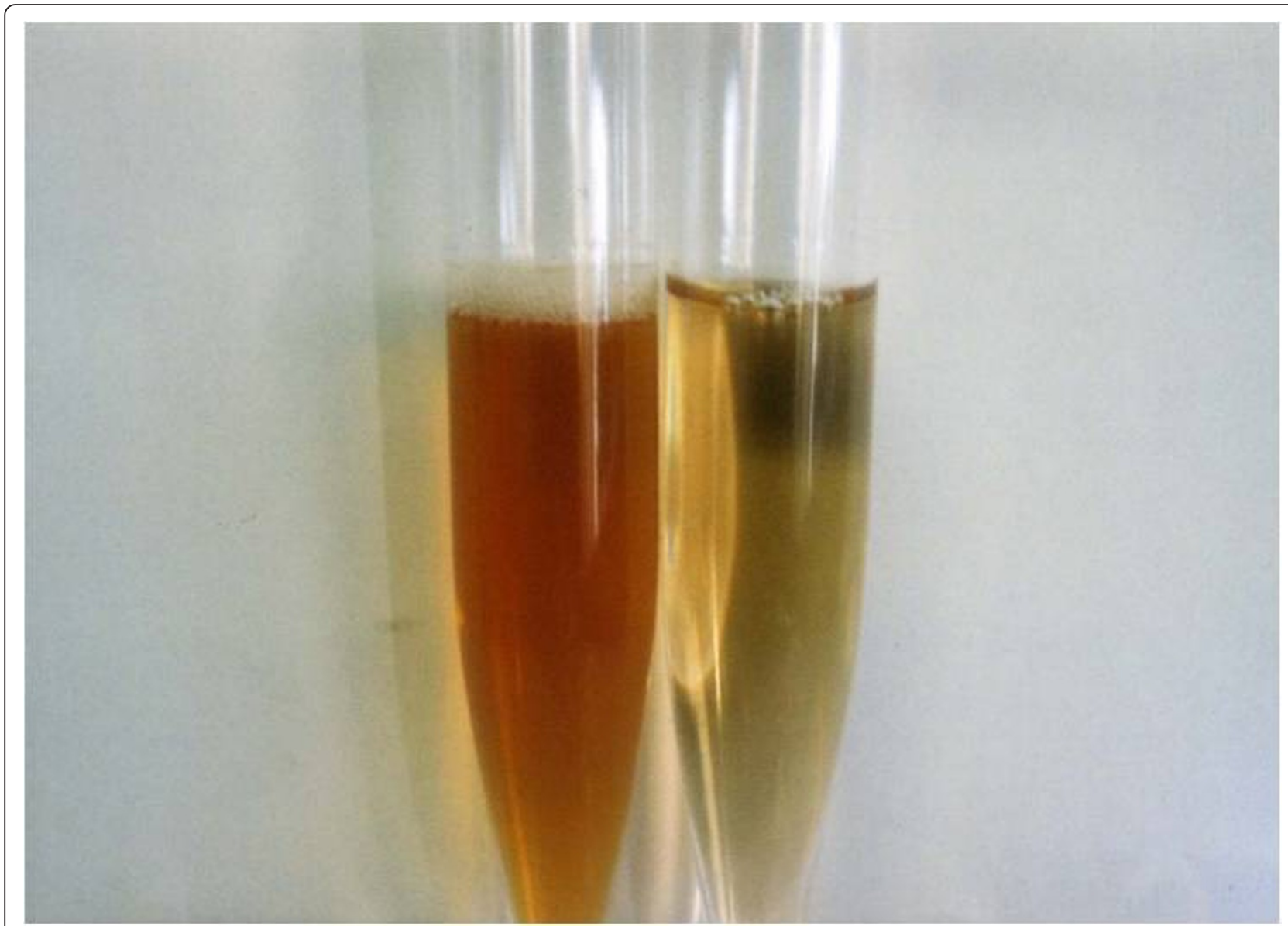

Figure 1 Patient's urine (left) and control urine (right). The patient's urine was red because of hemolysis. 
that results in different degrees of pancytopenia. The triad of hemolytic anemia, thrombosis, and pancytopenia makes PNH a truly unusual clinical syndrome [5]. These manifestations were visible in our case.

Deficient expression of CD55 and CD59 has recently been reported in patients with autoimmune hemolytic anemia, autoimmune thrombocytopenia, or SLE [6,7]. An autoimmune condition such as SLE may contribute to the pathogenesis of PNH [8]. It is a very interesting phenomenon and might be associated with the pathogenesis of our present case.

\section{Conclusions}

To the best of our knowledge, SLE with PNH is very rare and its mechanism is unknown. Clinicians should be aware of the association between autoimmune disease and $\mathrm{PNH}$.

\section{Consent}

Written informed consent was obtained from the patient for publication of this case report and any accompanying images. A copy of the written consent is available for review by the Editor-in-Chief of this journal.

\section{Abbreviations}

dsDNA: double-stranded DNA; LDH: lactate dehydrogenase; PNH: paroxysmal nocturnal hemoglobinuria; SLE: systemic lupus erythematosus.

\section{Author details}

Community Medicine, Hirosaki University Graduate School of Medicine, 5

Zaifu-cho, Hirosaki-city, Aomori, 036-8562, Japan. ${ }^{2}$ Department of

Nephrology, Hirosaki University School of Medicine, 5 Zaifu-cho, Hirosaki-city, Aomori, 036-8562, Japan

\section{Authors' contributions}

NN wrote the manuscript and was a treating physician for the patient. TS, KS, RK, MN, MS, TF, and RM were also treating physicians for the patient. YS and $\mathrm{HO}$ performed the literature search and helped to write the manuscript. $\mathrm{HY}$ and $\mathrm{KO}$ were the major contributors to the writing of the manuscript. All authors read and approved the final manuscript.

\section{Competing interests}

The authors declare that they have no competing interests.

Received: 8 June 2011 Accepted: 14 November 2011

Published: 14 November 2011

\section{References}

1. Rotoli B, Luzzatto L: Paroxysmal nocturnal hemoglobinuria. Semin Hematol 1989, 26:201-207.

2. Hochberg MC: Updating the American College of Rheumatology revised criteria for the classification of systemic lupus erythematosus. Arthritis Rheum 1997, 40:1725.

3. Schwartz RS: The target gene in paroxysmal nocturnal hemoglobinuria. $N$ Engl J Med 1994, 330:283-284.

4. Richard SJ, Rawstron AC, Hillmen P: Application of flow cytometry to the diagnosis of paroxysmal nocturnal hemoglobinuria. Cytometry 2000, 42:223-233.

5. Bessler M, Hillmen P: Somatic mutation and clonal selection in the pathogenesis and in the control of paroxysmal nocturnal hemoglobinuria. Semin Hematol 1998, 25:149-167.
6. Ruiz-Arguelles A, Llorente $L$ : The role of complement regulatory proteins (CD55 and CD59) in the pathogenesis of autoimmune hemocytopenias. Autoimmun Rev 2007, 6:155-161.

7. Ruiz-Delgado GJ, Vázquez-Garza E, Méndez-Ramírez N, Gómez-Almaquer D: Abnormalities in the expression of CD55 and CD59 surface molecules on peripheral blood cells are not specific to paroxysmal nocturnal hemoglobinuria. Hematology 2009, 14:33-37.

8. Luzzatto L, Gianfaldoni G: Recent advances in biological and clinical aspects of paroxysmal nocturnal hemoglobinuria. Int J Hematol 2006, 84:104-112.

doi:10.1186/1752-1947-5-550

Cite this article as: Nakamura et al:: Paroxysmal nocturnal hemoglobinuria in systemic lupus erythematosus: a case report. Journal of Medical Case Reports 2011 5:550.

\section{Submit your next manuscript to BioMed Central and take full advantage of:}

- Convenient online submission

- Thorough peer review

- No space constraints or color figure charges

- Immediate publication on acceptance

- Inclusion in PubMed, CAS, Scopus and Google Scholar

- Research which is freely available for redistribution 